\title{
Effects of pore modification on the templating of guest molecules in a 2D honeycomb network
}

\author{
Minna T. Räisänen, ${ }^{a, b}$ Anna G. Slater (née Phillips), ${ }^{c}$ Neil R. Champness*c and Manfred Buck*a \\ Received (in $X X X, X X X)$ Xth $X X X X X X X X X 20 X X$, Accepted $X$ th $X X X X X X X X X 20 X X$ \\ ${ }_{5}$ DOI: 10.1039/b000000x
}

1,7-diadamantanethioperylene-3,4:9,10-tetracarboxylic diimide, (Ad-S) ${ }_{2}-\mathrm{PTCDI}$, adsorbed on $\mathrm{Au}(111)$

from solution was investigated by scanning tunneling microscopy (STM) and X-ray photoelectron

spectroscopy (XPS). (Ad-S) $)_{2}$-PTCDI forms a well-ordered monolayer whose structure is described by a

$(2 \sqrt{ } 63 \times 2 \sqrt{ } 19) \mathrm{R} 19.1^{\circ}$ chiral unit cell containing four molecules. Codeposition of (Ad-S $)_{2}-\mathrm{PTCDI}$ with

10 1,3,5-triazine-2,4,6-triamine (melamine) yields a honeycomb network whose $(7 \sqrt{3} \times 7 \sqrt{3}) \mathrm{R} 30^{\circ}$ unit cell is identical to the unsubstituted PTCDI/melamine analogue. The effect of the adamantyl thioether moieties on the adsorption of guest molecules is investigated using adamantane thiol and $\mathrm{C}_{60}$. While the thioether units do not affect the packing of adamantane thiol molecules a pronounced influence is seen in the case of fullerene. Pore modification involving different combinations of enantiomers of (Ad-S $)_{2}-\mathrm{PTCDI}$ give

15 rise to distinctly different arrangements of $\mathrm{C}_{60}$ molecules. The diversity of patterns is further increased by the presence of unsubstituted PTCDI molecules.

\section{Introduction}

Two dimensional supramolecular networks, prepared on different substrates both by vapour deposition in an ultrahigh vacuum 20 (UHV) environment and from solution, can be exploited as templates to direct the adsorption of guest entities. ${ }^{1-8}$ As long as periodicity, pore area, geometry and chemical functionality can be varied independently these systems provide an unprecedented precision and flexibility in the control over arrangement of guest 25 entities. However, since supramolecular networks rely on relatively weak non-covalent interactions and are usually determined by a balance of different interactions, the possibility of a change in the overall structure upon modification of a building block is inherent to these systems, thus making 30 systematic tuning of structures and properties challenging. While this is particularly pronounced for one component systems as highlighted by derivatives of perylene-3,4:9,10tetracarboxydiimide (PTCDI) ${ }^{9}$ bicomponent systems might be more tolerant provided that the synthon involves sufficiently 35 strong directional interactions such as the triple hydrogen bonding between melamine and PTCDI. ${ }^{9}$

As another step towards the controlled design of supramolecular structures we focus here on the aspect of pore modification by investigating how variations of the inner structure of a pore 40 affects the incorporation of guest molecules. A bimolecular PTCDI/melamine network was chosen which has been studied previously ${ }^{10-17}$ and proven to be sufficiently robust to act as a flexible template in solution based processing. ${ }^{15}$ Extending previous studies of bromine and propylthio (PrS) substituted 45 PTCDI derivatives ${ }^{9,18,19}$ the effect of the sterically bulky adamantyl thioether appendage is investigated here. Compared to the smaller $\mathrm{Br}$ and the flat lying $\mathrm{PrS}$ moiety, the three dimensional structure of adamantane is expected to exert a significantly different influence on the arrangement of guest 50 molecules. The adamantyl unit is attached to the PTCDI core on opposite sides in the so-called bay regions (Fig. 1). This substitution breaks the mirror symmetries of the PTCDI molecule and as a consequence (Ad-S) $)_{2}-$ PTCDI becomes chiral when adsorbed on a surface. If (Ad-S $)_{2}-$ PTCDI behaved like the 55 unsubstituted molecule codeposition with melamine ${ }^{9,15,16,18}$ would yield a honeycomb network whose pores are modified by the adamantyl moieties, as schematically shown in Fig. 1. Since the adamantyl moieties are well separated the pore area available for guest molecules will be critically dependent on their size.

${ }_{60}$ For this reason we have chosen two guest molecules which differ substantially in size. One is $\mathrm{C}_{60}$ which is also of general interest due to its physical properties. ${ }^{20,21}$ However, due to the high mobility of fullerenes highly ordered surface structures are difficult to achieve at room temperature. Thus in the context of ${ }_{65}$ host-guest surface chemistry ${ }^{7,9-14,22-30}$ the porous networks offer a valuable strategy for precisely controlling the arrangement, intermolecular distances, and interactions of fullerene molecules. ${ }^{20,31,32}$

The other guest molecule is adamantane thiol (AdSH). With a 70 van der Waals radius of approximately $3.5 \AA^{33}$ it is significantly smaller than the $10 \AA$ sized $\mathrm{C}_{60}{ }^{34,35}$ and it can be expected that the effect of the pore modification is substantially different for the two molecules.

\section{Experimental}

75 All starting materials were used as received unless otherwise stated. Perylene-3,4:9,10-tetracarboxylic acid (Aldrich) was 


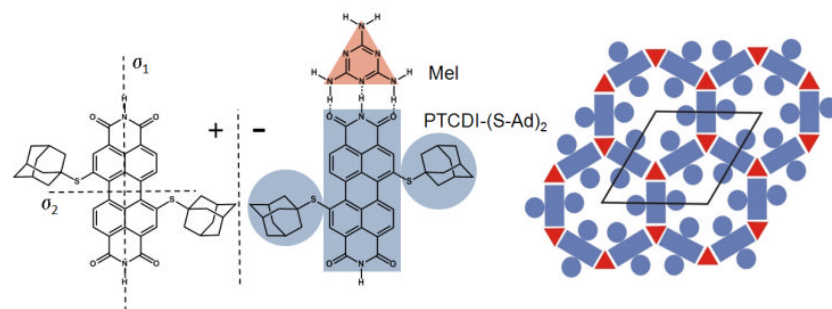

Fig. 1 Mirror images (+/-) of the structure of 1,7-diadamantane thioperylene-3,4:9,10-tetracarboxydiimide, (Ad-S) $)_{2}-\mathrm{PTCDI}$, and the 5 motif of the hydrogen bonding between the PTCDI derivative and melamine (Mel). The bimolecular network with the adamantylfunctionalised pores is shown on the right together with the unit cell.

brominated according to a literature procedure ${ }^{17}$ and converted to $N, N$ '-bis(2-ethylhexyl)dibromoperylene tetracarboxylicdiimide 10 with 2-ethylhexylamine in butanol/ $\mathrm{H}_{2} \mathrm{O} .{ }^{18}$ All reactions were carried out under an atmosphere of nitrogen. Column chromatography was performed on silica gel (Merck silica gel 60, 0.2-0.5 mm, 50-130 mesh). ${ }^{1} \mathrm{H}$ NMR and ${ }^{13} \mathrm{C}$ NMR spectra were obtained on a Bruker $300 \mathrm{MHz}$ spectrometer. Microanalyses 15 were performed by Stephen Boyer, London Metropolitan University. MS spectra (MALDI-TOF-MS) were determined on a Voyager-DE-STR mass spectrometer.

\section{Synthesis}

N'N-bis(2-ethylhexyl)-1,7-di(adamantanethio)perylene-3,4:9, 1020 tetracarboxylic diimide: A $250 \mathrm{~mL}$ round-bottomed flask was flame-dried, backfilled with nitrogen three times and charged with dry toluene $(100 \quad \mathrm{~mL}) . \quad N, N^{\prime}$-bis(2ethylhexyl)dibromoperylene tetracarboxylicdiimide (1 g, 1.29 mmol) and 1-adamantanethiol (0.65 g, $3.88 \mathrm{mmol})$ were added 25 and the mixture was heated at $80{ }^{\circ} \mathrm{C}$ under $\mathrm{N}_{2}$ for $1 \mathrm{~h}$. ${ }^{\mathrm{t}} \mathrm{BuONa}$ $(0.25 \mathrm{~g}, 2.59 \mathrm{mmol})$ and $\mathrm{Pd}\left(\mathrm{PPh}_{3}\right)_{4}(0.15 \mathrm{~g}, 5 \mathrm{~mol} \%)$ were then added and the mixture was heated at $90{ }^{\circ} \mathrm{C}$ under $\mathrm{N}_{2}$ overnight. The toluene was removed in vacuo, and the resulting purple residue was dissolved in chloroform $(200 \mathrm{~mL})$ and filtered by 30 gravity before washing with water $(3 \times 100 \mathrm{~mL})$. The organic fractions were dried with $\mathrm{Na}_{2} \mathrm{SO}_{4}$, filtered by gravity and evaporated in vacuo to yield a dark purple solid, which was purified via column chromatography $\left(\mathrm{SiO}_{2}, \mathrm{CHCl}_{3}\right)$ to yield a dark purple solid $(0.73 \mathrm{~g}, 60 \%)$. ${ }^{1} \mathrm{H}$ NMR $\left(400 \mathrm{MHz}, \mathrm{CDCl}_{3}\right) \delta_{\mathrm{H}}$ ${ }_{35}=9.42(\mathrm{~d}, J=8.03 \mathrm{~Hz}, 2 \mathrm{H}) 8.93(\mathrm{~s}, 2 \mathrm{H}) 8.68(\mathrm{~d}, J=8.16 \mathrm{~Hz}, 2 \mathrm{H})$ 4.07 - 4.27 (m, 4 H) 2.08 (br. s.) 1.84 (m) 1.22 - 1.74 (m) 0.99 (t, $J=7.40 \mathrm{~Hz}, 6 \mathrm{H}) 0.91(\mathrm{t}, J=7.03 \mathrm{~Hz}, 6 \mathrm{H}) .{ }^{13} \mathrm{C} \mathrm{NMR}(126 \mathrm{MHz}$, $\left.\mathrm{CDCl}_{3}\right) \delta_{\mathrm{C}}=163.93,163.77,141.54,139.31,134.05,131.95$, $130.79,129.85,128.35,127.28,122.58,120.52,53.51,44.39$,

40 43.46, 38.02, 35.81, 30.74, 30.05, 28.68, 24.09, 23.11, 14.10, 10.63. MS (MALDI-TOF): M/z 946.5 (M). EA: Calculated (\%): C 76.07 H 7.45 N 2.96 Found (\%): C 75.97 H 7.54 N 2.88.

1,7-di(adamantanethio)perylene-3,4:9,10-tetracarboxylic dianhydride: $\quad \mathrm{N}, \mathrm{N}$ '-bisdiethylhexyl-1,7-diadamantanethio45 perylene-3,4,9,10-tetracarboxylicdiimide (100 mg, $0.105 \mathrm{mmol})$ was suspended in iso-propanol $(50 \mathrm{~mL})$ and sonicated for 15 minutes at RT, giving a purple solution. $\mathrm{KOH}$ (592 mg, 10.5 mmol) was then added and the suspension stirred at RT overnight. The resulting bright orange solution was then poured 50 into a solution of $50 \%$ glacial acetic acid/water $(50 \mathrm{~mL})$ and stirred for $1 \mathrm{~h}$ at RT. A pink solid precipitated and was collected by vacuum filtration, washed with water and dissolved in chloroform $(50 \mathrm{~mL})$. The purple solution was reduced in vacuo and the resulting purple solid used without further purification. ${ }_{55}$ MS (MALDI-TOF): M/z 724.2 (M).

1,7-di(adamantanethio)perylene-3,4:9,10-tetracarboxylic diimide $(A d-S)_{2}-P T C D I: \quad$ 1,7-diadamantanethiolperylene-3,4,9,10tetracarboxylicdianhydride $(50 \mathrm{mg}, 0.07 \mathrm{mmol})$ was dissolved in THF $(25 \mathrm{~mL})$ and placed in a flask with ammonia (40 mL, $35 \%)$. ${ }_{60}$ This was heated at $50{ }^{\circ} \mathrm{C}$ for $18 \mathrm{~h}$, yielding a dark purple precipitate. This suspension was cooled to room temperature and poured over ice, whereupon glacial acetic acid was added dropwise to neutralise excess ammonia. The precipitate was collected by vacuum filtration, washed with water and dissolved 65 in chloroform $(50 \mathrm{~mL})$ before being reduced in vacuo to yield a purple solid. The resulting solid was purified via column chromatography $\left(\mathrm{SiO}_{2}, \mathrm{CHCl}_{3} / 0.5 \% \mathrm{MeOH}\right)$ to yield a dark blue powder (40 mg, $80 \%$ ). MS (MALDI-TOF): M/z 722.2 (M).

\section{Sample preparation and characterisation}

$70 \mathrm{Au} /$ mica substrates (300 nm gold; Georg Albert PVD) were flame-annealed before sample preparation. (Ad-S $)_{2}-$ PTCDI SAMs were prepared by immersing $\mathrm{Au}(111)$ substrate into a hot (348-373 K) DMF solution of (Ad-S) $)_{2}-$ PTCDI $(210 \mu \mathrm{M}$ or 1 $\mathrm{mM}$ ) for 1 min after which the sample was rinsed with DMF and

75 dried under a flow of $\mathrm{N}_{2}$. The (Ad-S $)_{2}-\mathrm{PTCDI} /$ melamine network was prepared by modifying the previously reported procedure used for preparation of the unsubstituted PTCDI-melamine network [28]. Saturated DMF solution of melamine and $1 \mathrm{mM}$ DMF solution of (Ad-S) $)_{2}-\mathrm{PTCDI}$ were mixed in DMF to result in ${ }_{80} 1: 11$ and 1:80 dilutions, respectively. The mixture was then heated in an oven at $348 \mathrm{~K}$ for $30 \mathrm{~min}$ before the Au substrate was immersed into it for $1 \mathrm{~min}$ at the elevated temperature. After immersion the sample was dipped into DMF at room temperature and blown dried with $\mathrm{N}_{2}$.

${ }_{85}$ For obtaining a hybrid structure with thiol SAM, (Ad-S) $)_{2}-$ PTCDI /melamine modified $\mathrm{Au}$ substrate was immersed into $400 \mu \mathrm{M}$ 1,3,5-trichlorobenzene solution of adamantane thiol for $90 \mathrm{~s}$ after which the sample was rinsed with EtOH and blown dried with $\mathrm{N}_{2}$. (Ad-S $)_{2}-\mathrm{PTCDI} /$ melamine and PTCDI-melamine networks were 90 exposed to $\mathrm{C}_{60}$ at room temperature by immersion of the network modified $\mathrm{Au}(111)$ substrates into a 1,3,5-trichlorobenzene solution of $\mathrm{C}_{60}$ for $20 \mathrm{~s}$ after which they were blown dried with $\mathrm{N}_{2}$.

STM images were recorded under ambient conditions with a 95 PicoScan (Molecular Imaging) microscope and tips prepared by mechanically cutting a $\mathrm{Pt} / \mathrm{Ir}(80: 20)$ wire $(0.25 \mathrm{~mm}$ diameter, Advent Research Materials Ltd.). The STM data was collected in constant current mode.

XPS spectra were recorded with Thermo VG Scientific Sigma 100 Probe spectrometer at $9.6 \times 10^{-9}$ torr. A monochromatic $\mathrm{Al} \mathrm{K} \mathrm{K}_{\alpha} \mathrm{X}$ ray source was applied for all measurements with an analytical spot size of $0.5 \mathrm{~mm}^{2}$, a $37^{\circ}$ takeoff angle, $40 \mathrm{~ms}$ dwell time, and pass energies of $40 \mathrm{eV}$ ( $\mathrm{S} \mathrm{2p}, \mathrm{Au} 4 \mathrm{f}, \mathrm{C} 1 \mathrm{~s}, \mathrm{O} 1 \mathrm{~s}$ and $\mathrm{N} \mathrm{1s}$ ). Samples were referenced against the internal $\mathrm{Au} 4 \mathrm{f}_{7 / 2}$ line at 84.0 $105 \mathrm{eV}$. Data evaluation was carried using CasaXPS (Casa software Ltd).

\section{Results and Discussion}



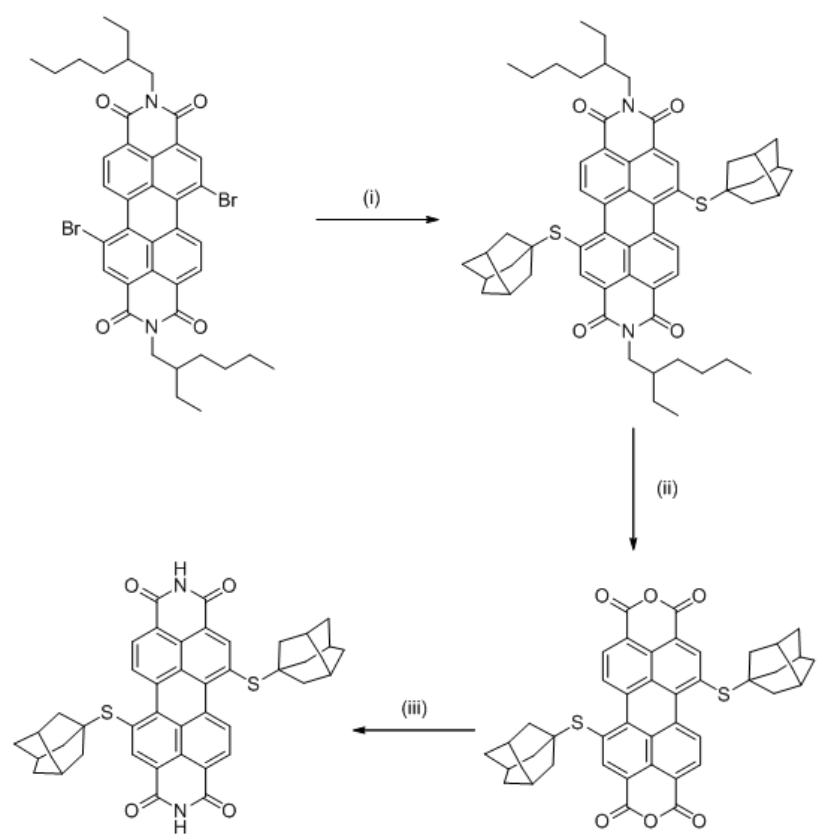

Scheme 1 Synthetic pathway used to prepare (Ad-S) ${ }_{2}-\mathrm{PTCDI}$. (i) AdSH, ${ }^{\mathrm{t}} \mathrm{BuONa}, \mathrm{Pd}\left(\mathrm{PPh}_{3}\right)_{4}$, toluene, $80-90^{\circ} \mathrm{C}, 24 \mathrm{~h}$,; (ii) $\mathrm{KOH},{ }^{\mathrm{i}} \mathrm{PrOH}, \mathrm{RT}, 24 \mathrm{~h}$; (iii) $\mathrm{NH}_{4} \mathrm{OH}, \mathrm{THF}, 50^{\circ} \mathrm{C}, 24 \mathrm{~h}$.

5 (Ad-S $)_{2}-$ PTCDI was prepared following the synthetic route shown in Scheme 1 in good overall yield. Introduction of the adamantylthioether unit to the perylene core was found to be most effective using a $\mathrm{Pd}\left(\mathrm{PPh}_{3}\right)_{4}$ catalysed coupling reaction between AdSH and $\mathrm{Br}_{2}$-PTCDI. Attempts to prepare (Ad-S $)_{2}$ ${ }_{10}$ PTCDI in a method analogous to that used to prepare $(\mathrm{PrS})_{2}-$ PTCDI, via reaction of $\mathrm{PrSH}$ with $\mathrm{Br}_{2}$-PTCDI in the presence of base, ${ }^{9}$ were unsuccessful.

\section{Monolayers of (Ad-S) $)_{2}-$ PTCDI}

Adsorption of (Ad-S) $)_{2}-\mathrm{PTCDI}$ at temperatures ranging between 15 348-373 K results in well ordered monolayers as illustrated by the STM image shown in Fig. 2a. The Fourier transform, Fig. 2a, reveals domains which are not in line with the symmetry axes of the substrate but which are aligned along directions differing by about $40^{\circ}$. In the magnified images (Fig. 2b) of the two domains 20 labelled by the dashed and solid lines in Fig. 2a two clearly separated alternating groups of four protrusions each (highlighted by red and blue circles) are discernible which yield mirror domains. The distances between the protrusions of a group are too small for them to be identified with PTCDI moieties, i.e., the 25 protrusions represent adamantyl units whereas the PTCDI moieties are not seen. The adamantyl units of a group are assigned to different (Ad-S) $)_{2}-$ PTCDI molecules which means that the PTCDI units lie between protrusions of the two different groups.

$30 \mathrm{~A}$ model describing all experimental observations is depicted in Fig. 2c. This structure is described by the matrices

$\left(\begin{array}{cc}18 & -6 \\ 4 & 6\end{array}\right)$ and $\left(\begin{array}{cc}12 & 6 \\ 10 & -6\end{array}\right)$

corresponding to oblique $(2 \sqrt{6} 63 \times 2 \sqrt{ } 19)$ unit cells containing four molecules and whose lattice vectors $\boldsymbol{a}(45.9 \AA)$ and $\boldsymbol{b}(25.2 \AA)$ are 35 rotated by $\mp 19.1^{\circ}$ and $\pm 36.6^{\circ}$ with respect to the $\langle 1 \overline{1} 0\rangle$ direction. We note that while these dimensions are based on the average of several STM images, deviations by about $\pm 5 \%$ from
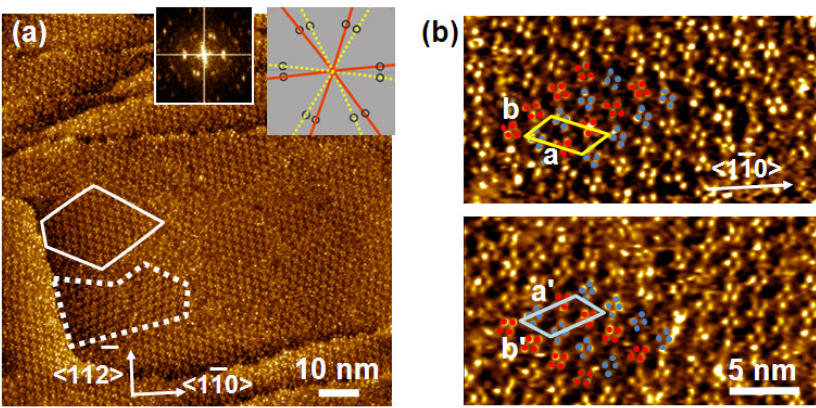

(c)

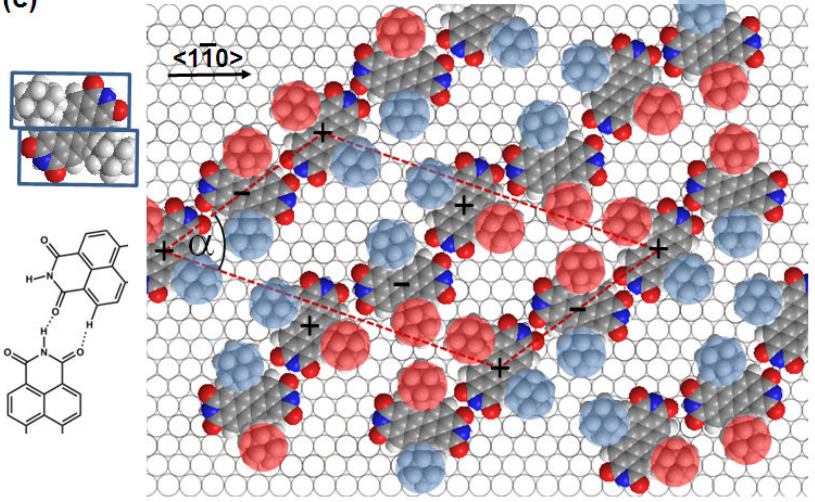

Fig. 2 a) Large-scale STM image of (Ad-S)2-PTCDI on Au(111). The 40 dotted and dashed lines frame two mirror domains which are also reflected in the Fourier transform (insets). The relationship between domains and diffraction spots is illustrated by the cartoon. $\mathrm{I}_{\mathrm{t}}=174 \mathrm{pA}$ $\mathrm{V}_{\text {bias }}=180 \mathrm{mV}$ b) Magnified images of the two domains marked in (a). Highlighted by red and blue circles are two groups of protrusions 45 identified as the adamantyl moieties. c) Model of the chiral (Ad-S)2PTCDI monolayer structure which is described by an oblique $(2 \sqrt{ } 63 \times 2 \sqrt{ } 19) \mathrm{R} 19.1^{\circ}$ unit cell $\left(\mathrm{a}=45.9 \AA, \mathrm{b}=25.2 \AA, \alpha=55.7^{\circ}\right)$. The projected area of the space filling structure by two rectangles is shown on the left (for details see text).

50 these dimensions are within the error margins of the experimental measurements. The monolayer structure is described by zig-zag rows of (Ad-S) $)_{2}-$ PTCDI molecules with the PTCDI units interacting via the hydrogen bonding motif depicted in Fig. 2c. The unit cell is chiral but, similar to other cases such as 55 heptahelicene, ${ }^{36}$ contains a racemic mixture of both enantiomers. While there is some uncertainty about the exact positioning of the adamantyl units relative to the PTCDI core due to the unknown angles associated with the C-S-C groups for the molecule adsorbed on $\mathrm{Au}$, placement of the adamantyl units on the mirror ${ }_{60}$ axis $\sigma_{2}$ (for definition see Fig. 1) can be excluded as this would be in conflict with the steric requirements of the PTCDI core and the observed distances between the adamantyl units.

Comparing the size per (Ad-S) $)_{2}$-PTCDI molecule of about 160 $\AA^{2}$, using a simple geometric approximation, as illustrated in Fig. ${ }_{65} 2 \mathrm{c}$, with the area per molecule of about $240 \AA^{2}$ calculated from the unit cell size shows that the coverage is well below a dense packing. The formation of this rather open structure which we consistently observed irrespective of the preparation conditions seems surprising considering that the substantial adsorption 70 energy of perylene derivatives which is expected to be in the range of $2 \mathrm{eV}^{37}$ should favour a maximisation of coverage, even though the value for (Ad-S) $)_{2}-\mathrm{PTCDI}$ might deviate to some extent due to the non-flat geometry of the free molecule and additional interactions of the sulfur with the gold substrate. The 75 more open structure suggests a balance of contributions which, in addition to the adsorption energy comprises directional $\mathrm{H}$ bonding interaction and interactions between the adamantyl units. 
Compared to analogous compounds where, instead of adamantyl thioether groups, the PTCDI unit is substituted by bromine, ${ }^{9}$ $\operatorname{PrS}^{9,19}$ or benzoic acid ${ }^{18}$ the structure observed here is different. In contrast to the propyl thioether analogue where the 5 intermolecular $\mathrm{C}-\mathrm{H}$... O interaction of the alkane chain with the carbonyl group of PTCDI yields a porous hexagonal structure, this type of interaction does not seem to be of decisive influence in the case of (Ad-S $)_{2}-\mathrm{PTCDI}$, perhaps precluded by the rigidity of the adamantyl moiety. It is also noted that the $\mathrm{C}-\mathrm{H}$... O 10 interaction of propyl thioether results in an enantiomerically pure structure, within given islands, whereas (Ad-S $)_{2}-\mathrm{PTCDI}$ seems to consist of a racemic mixture. It is also different from the hexagonal pattern of the benzoic acid derivative ${ }^{18}$ and the one dimensional structure of the bromine substituted PTCDI. ${ }^{9}$ The 15 fact that all four substituted PTCDI molecules form different structures clearly demonstrates that the intermolecular hydrogen bonding between the imide units is not sufficiently strong to allow for a systematic variation of a basic pattern such as the hexagonal structure. The subtle balance between different 20 contributions to the free energy of the system is further highlighted by the change in structure from PTCDI to the dianhydride analogue. For the PrS substituted perylenetetracarboxylic dianhydride (PTCDA) a dense molecular packing is found ${ }^{38}$ in agreement with the weaker interaction and, 25 thus, small directional forces between anhydride compared to imide groups.

\section{Coadsorption of (Ad-S) $)_{2}-$ PTCDI and Melamine}

Provided the triple hydrogen bond between PTCDI and melamine is dominating the intermolecular forces, a honeycomb network 30 structure should form whose pore geometry is modified by the adamantyl unit as illustrated in Fig. 1. Following our previously described solution-based method ${ }^{15-17}$ (Ad-S) $)_{2}-$ PTCDI and melamine are co-deposited from solution. While it is not immediately obvious from the large scale real space image shown 35 in Fig. 3a, the well defined spots seen in the Fourier transform clearly demonstrates that a highly crystalline structure is formed. The apparent lack of order in the real space structure becomes even more obvious in the high magnification image of Fig. 3b, in particular when compared with the unsubstituted 40 PTCDI/melamine honeycomb structure shown in the inset of Fig. 3b. The discrepancy between real space image and the Fourier transform becomes clear when analysing the structure in more detail. The upper part of Fig. $3 \mathrm{c}$ is a cutout from Fig. $3 \mathrm{~b}$ marked by the rectangle. With the aid of the Fourier filtered image shown 45 in the bottom part of Fig. 3c the network structure can be easily identified with dark regions corresponding to the melamine vortices of the network. Superimposing this network structure onto the unfiltered STM image reveals that the protrusion marked by the red circles exhibit a pattern. In the majority of cases the ${ }_{50}$ protrusions are paired and can be straightforwardly assigned to the adamantyl moieties of PTCDI compound. The hexagonal honeycomb structure is now evident and demonstrates that, in contrast to the pure (Ad-S) $)_{2}$-PTCDI layer hydrogen bonding now plays the decisive role in structure formation.

${ }_{55}$ The difficulty in identifying a regular structure in the real space image can be explained by two factors. Firstly a dynamic effect causes variations in the apparent height of the protrusions. Taking into account that the adamantyl units are well separated from each other some residual conformational flexibility can be 60
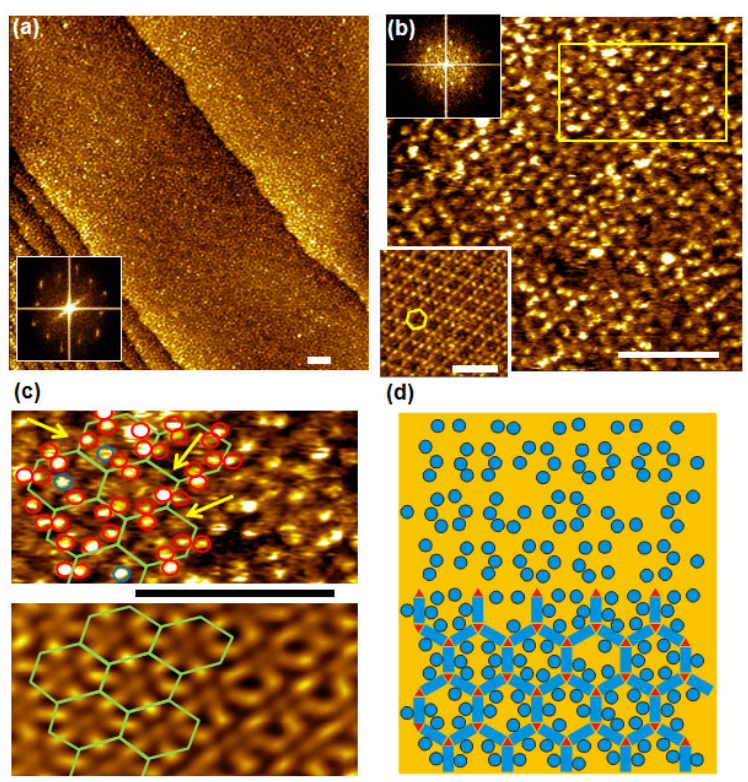

(d)

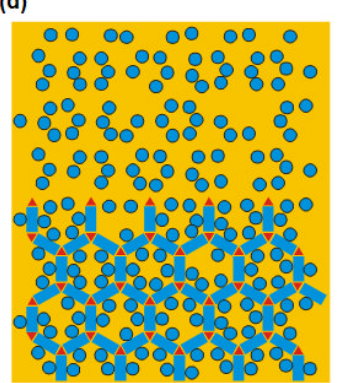

Fig. 3 STM images of (Ad-S $)_{2}-\mathrm{PTCDI}$ and melamine co-deposited on $\mathrm{Au}(111)$ from DMF solution. $I_{\text {set }}=15 \mathrm{pA}, V_{\text {bias }}=680 \mathrm{mV}$. All scale bars 10 nm. a,b) Large-scale (a) and high resolution images (b). Fourier 65 transforms are shown as insets. For comparison a PTCDI-melamine network is shown in the lower left corner of b). c) Enlarged section of surface marked by the rectangle in b), showing data as acquired (top) and Fourier filtered (bottom). d) Model of the bimolecular network formed by (Ad-S $)_{2}-$ PTCDI and melamine with some adamantyl units missing. The 70 apparent lack of order is illustrated in the top half where the PTCDI and melamine units have been omitted.

expected to result in a dynamic behaviour when the adamantyl units experiences the field of the scanning tip. The second reason is static in nature and arises from defects in the network. Marked 75 by the arrows in Fig. 3c, some of the PTCDI sites show either only one protrusion or none at all. Furthermore, some protrusions as highlighted by the blue circles appear to be detached from the PTCDI units. This suggests that under the conditions of network preparation the thioether link is subject to some degradation, i.e., 80 the adamantyl moiety can be detached from the perylene core but remains on the surface as adamantyl-thiolate. This explains the presence of both dislocated protrusions and PTCDI network units with one or even two adamantyl units missing. While the latter might also be explained by the dynamic imaging effect, the fact 85 that it is not changing between scans is a strong indication of defects. The definite proof of the detachment of the thioadamantyl units is provided below when discussing the adsorption of $\mathrm{C}_{60}$ into the network pores.

Within the precision of our STM measurements the dimensions 90 and orientation of the adamantyl modified network is found to be identical to the structure of unsubstituted PTCDI, i.e., it has a periodicity of $35 \AA$ and a $(7 \sqrt{ } 3 \times 7 \sqrt{ } 3) \mathrm{R} 30^{\circ}$ unit cell indicated in Fig. 1. A close look at the exact position of the adamantyl units in Fig. 3c shows a narrow distribution in position. Even though 95 there are cases where, within the resolution limit, the adamantyl moieties seem to be positioned symmetrically on the $\sigma_{2}$ axis of the PTCDI core and in this case STM is unable to differentiate between different enantiomers. Mostly two adamantyl units 
attached to the same PTCDI core are on opposite sides of the $\sigma_{2}$ mirror axis with both possible configurations occurring consistent with the presence of $(+)$ and $(-)$ enantiomers. This means that unless a pore consists of an enantiomerically pure arrangement of $5(\mathrm{Ad}-\mathrm{S})_{2}-\mathrm{PTCDI}$ molecules there is a substantial variation in the distance between adjacent adamantyl units of a pore depending on whether the (Ad-S $)_{2}-$ PTCDI molecules of the same or opposite chirality are next to each other. This affects the adsorption of guest molecules as will be shown below. The 10 experimental observations for the empty (Ad-S $)_{2}$ PTCDI/melamine network are summarised in the cartoon of Fig. 3d. As experimentally observed, some of the adamantyl units are omitted from the figure and it can be seen that, in the absence of the honeycomb lattice, it is difficult to recognise the regular 15 arrangement formed by the structure.

The formation of analogous bimolecular honeycomb networks by $\mathrm{Br}$, and PrS substituted analogues as well as (Ad-S $)_{2}-\mathrm{PTCDI}$ in combination with melamine, contrasts with the large variation in structures observed for monomolecular layers of the various 20 substituted PTCDI compounds. The predictability in forming the bimolecular honeycomb networks evidences that the triple hydrogen bond formed between PTCDI and melamine is the dominating structural element in directing the bimolecular structure. Indeed, the imide-melamine interaction is sufficiently 25 robust to allow for systematic variation of the network.

Even though the (Ad-S $)_{2}-$ PTCDI containing network results in a pronounced modification of the pore geometry, the presence of unsubstituted and monosubstituted PTCDI molecules (Ad-S $)_{0,1}$ PTCDI) which are statistically distributed in the network at a 30 level of about $20 \%$ limits the degree of control over the pore geometry. While the exact origin of the imperfections remains unclear at present, one explanation for the presence of defective building blocks are impurities present in the (Ad-S) $)_{2}-\mathrm{PTCDI}$ compound. Even though the chemical analysis of the bulk 35 compound does not show a level of contamination above detection limit a massive enrichment on the surface during the formation of the bimolecular network could occur if the adsorption energies and/or the adsorption kinetics of the respective compounds are very different. ${ }^{39}$ However, since the

40 number of missing adamantyl protrusions in the STM images are very different for the monomolecular (Ad-S $)_{2}-$ PTCDI layer and the bimolecular network, a drastically different adsorption behaviour would be required for both systems. The other obvious explanation for the presence of imperfections is thermal 45 degradation. The problem here is that preparation of the monomolecular (Ad-S $)_{2}-$ PTCDI layer under conditions of the network does not increase the defects. Therefore, the difference between the two types of layers is explained by a pronounced dependence of the thermal stability of (Ad-S $)_{2}-$ PTCDI under the

50 experimental conditions used. While on the one hand studies of dialkylsulfides on gold excluded a cleavage of the C-S bond, ${ }^{39,40}$ a study of $n$-dioctadecyl sulfide found ${ }^{41}$ that cleavage is possible but strongly dependent on the preparation parameters such as temperature and, interestingly, also the choice of solvent. The

55 occurrence of isolated circular protrusions which are marked by the blue circles in Fig. 3c could be taken as an indication that $\mathrm{C}-\mathrm{S}$ bond cleavage indeed occurs, producing separate adamantyl thiolate species during the network preparation. Considering the similarity in the preparation conditions used for both bimolecular 60 and monomolecular arrays it is reasonable to conclude that the melamine acts as a cleavage promoting base. (a)

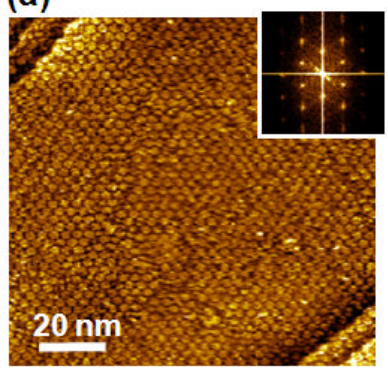

(b)

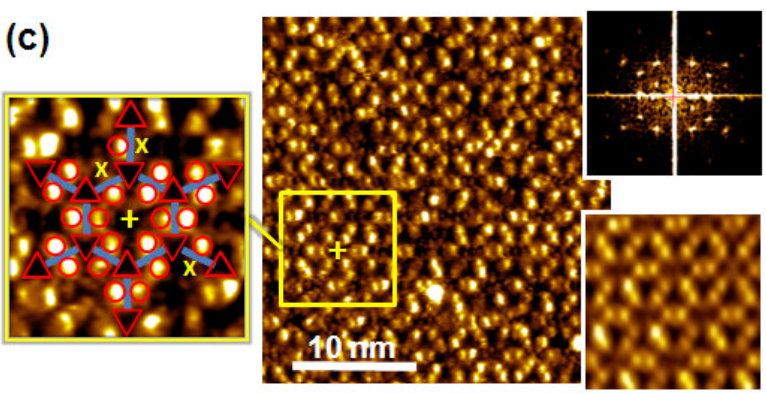

(d)

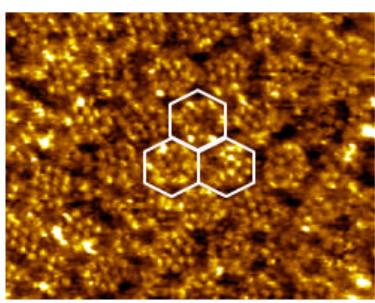

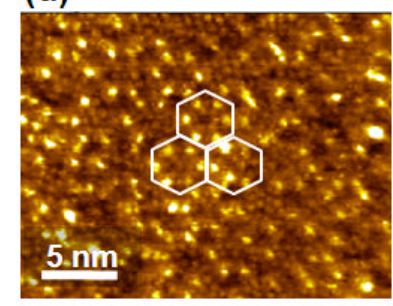

Figure 4. STM images of (Ad-S $)_{2}-\mathrm{PTCDI} /$ Melamine network with adamantane thiol, $\mathrm{Ad}_{\mathrm{G}}$, filled pores. a) Large scale image with Fourier 65 transform shown as inset. $\mathrm{U}_{\mathrm{b}}=-340 \mathrm{mV}$ and $\mathrm{I}_{\mathrm{t}}=36 \mathrm{pA}$. b) Molecularly resolved image with network frame indicated. $\mathrm{U}_{\mathrm{b}}=-340 \mathrm{mV}$ and $\mathrm{I}_{\mathrm{t}}=$ $174 \mathrm{pA}$. Inset shows magnified image of pore marked highlighted by the square. c) $\mathrm{AdSH}, \mathrm{Ad}_{\mathrm{G}}$, filled network imaged under different tunneling conditions. Fourier transform and Fourier filtered image are shown on the 70 right. Enlarged section of the network with structure overlaid depicted on the right. " $\mathrm{x}$ " mark adamantyl units missing from the (Ad-S) $)_{2}-\mathrm{PTCDI}$ moieties. $U_{b}=180 \mathrm{mV}$ and $\mathrm{I}_{\mathrm{t}}=92 \mathrm{pA}$. d) Illustration of contrast change upon change of tunneling current. Same area scanned at $\mathrm{U}_{\mathrm{b}}=-340 \mathrm{mV}$ and $\mathrm{I}_{\mathrm{t}}=45 \mathrm{pA}$ (left) and $174 \mathrm{pA}$ (right), respectively.

\section{${ }_{75}(\mathrm{Ad}-\mathrm{S})_{2}-\mathrm{PTCDI} / \mathrm{melamine}$ network as template}

To study the effect of pore modification by the adamantyl moieties, two molecules differing in size, namely adamantyl thiol and $\mathrm{C}_{60}$, were chosen as guest molecules. Both molecules have been shown to adsorb into pores ${ }^{14,15}$ and their rigid structure 80 facilitates imaging at molecular resolution.

\section{Adamantane thiol adsorption}

To avoid confusion between the adamantyl moieties attached to PTCDI and adamantane thiol guest molecules we refer to them in 85 the following as $\mathrm{Ad}_{\mathrm{N}}$ and $\mathrm{Ad}_{\mathrm{G}}$, respectively.

The large scale image shown in Fig. 4a, together with the Fourier transform, indicates that analogous to the PTCDI-melamine network $^{15}$ the adamantyl modified network is sufficiently stable to act as template for thiol adsorption from solution. This is fully 
confirmed by the high resolution image (Fig. 4b) where the $\mathrm{Ad}_{\mathrm{G}}$ molecules are discernible inside the pores. To guide the eye the network pattern is highlighted by the hexagons in the upper part of the image. The network is reflected by a hexagonal mesh of 5 depressions which arise from the melamine molecules as highlighted by the enlarged image of a pore shown in the inset. Unlike the unsubstituted PTCDI/melamine network, ${ }^{15}$ the (AdS) ${ }_{2}$-PTCDI analogue does not appear as a continuous hexagonal mesh of grooves. The presence of the $\operatorname{Ad}_{\mathrm{N}}$ units affects the 10 measured height above the PTCDI units, most likely due to the somewhat shorter distance between two adamantyl units of a (Ad-S $)_{2}-$ PTCDI molecule compared to two $\mathrm{Ad}_{\mathrm{G}}$ molecules adsorbed on either side of an unsubstituted PTCDI molecule. Otherwise there is no obvious difference between the present 15 network and the analogous network formed by unsubstituted PTCDI. In both cases 12-14 densely packed adamantyl units are observed with an intermolecular distance of around $7 \AA$ which agrees with the value of $6.9 \AA$ given for uniform adamantane thiol SAMs. ${ }^{33}$ This might not be too surprising considering the 20 identical nature of pore modifier and guest molecule. Furthermore, the distance between two adjacent $\operatorname{Ad}_{\mathrm{N}}$ moieties in a pore is sufficient for an $\mathrm{Ad}_{\mathrm{G}}$ molecule to fit in between.

Somewhat unexpected is the lack of height contrast between $\mathrm{Ad}_{\mathrm{N}}$ and $\mathrm{Ad}_{\mathrm{G}}$ units in the image shown in Fig. 4b. Despite their 25 similarity, $\operatorname{Ad}_{\mathrm{N}}$ is attached to PTCDI and interacts with the substrate via a thioether group whereas $\operatorname{Ad}_{\mathrm{G}}$ is adsorbed as a thiolate. Therefore, one could expect that differences in the bonding to the substrate is also reflected in the tunneling resistance. Indeed as Fig. $4 \mathrm{c}$ illustrates $\mathrm{Ad}_{\mathrm{N}}$ and $\mathrm{Ad}_{\mathrm{G}}$ can appear 30 very differently. A different set of tunneling parameters gives rise to a pronounced contrast between the two types of adamantyl moieties. The change from negative to positive bias highlights now $\mathrm{Ad}_{\mathrm{N}}$ whereas the $\mathrm{Ad}_{\mathrm{G}}$ molecules appear dark and cannot be resolved. In agreement with the results for the empty network, 35 some of the PTCDI moieties are missing an $\operatorname{Ad}_{\mathrm{N}}$ unit as demonstrated by the enlarged image of the area marked by the yellow square. Whereas most PTCDI units have two $\mathrm{Ad}_{\mathrm{N}}$ units (marked by red circles), some defects marked by " $\mathrm{x}$ " are discernible. It is interesting to note that, compared to the empty 40 network, imaging of the filled network under conditions where the $\mathrm{Ad}_{\mathrm{N}}$ moieties are highlighted gives a better resolved image of the adamantyl moieties. They seem to be better defined with regard to both variations in apparent height and position. This is also reflected by the Fourier filtered image where in the pore 45 filled network the $\operatorname{Ad}_{\mathrm{N}}$ units appear as separate protrusions, in contrast to the corresponding blurred image of the empty network (Fig. 3c). This can be rationalised by a reduced conformational flexibility of the $\operatorname{Ad}_{\mathrm{N}}$ units due to the presence of densely packed $\mathrm{Ad}_{\mathrm{G}}$ thiolates.

${ }_{50}$ That the contrast variation is indeed originating from the tunneling is evidenced by Fig. $4 \mathrm{~d}$ where images of the same area but acquired under different tunneling conditions are shown. The change from highlighted $\mathrm{Ad}_{\mathrm{N}}$ units (left) when imaged at lower currents to the same tunneling contrast of the $\operatorname{Ad}_{N}$ and $\operatorname{Ad}_{G}$ 55 moieties at higher currents demonstrates the possibility to discriminate between the two differently attached adamantyl units. However, while the tunneling parameters do change the relative heights of the $\mathrm{Ad}_{\mathrm{N}}$ and $\mathrm{Ad}_{\mathrm{G}}$ additional factors can also affect the contrast. Differences as shown in Fig. 4 can even occur 60 within a single scan, i.e. with tunneling parameters remaining unchanged, thus, evidencing that the state of the STM tip is also crucial for the imaging contrast. Since the tip state is crucial it
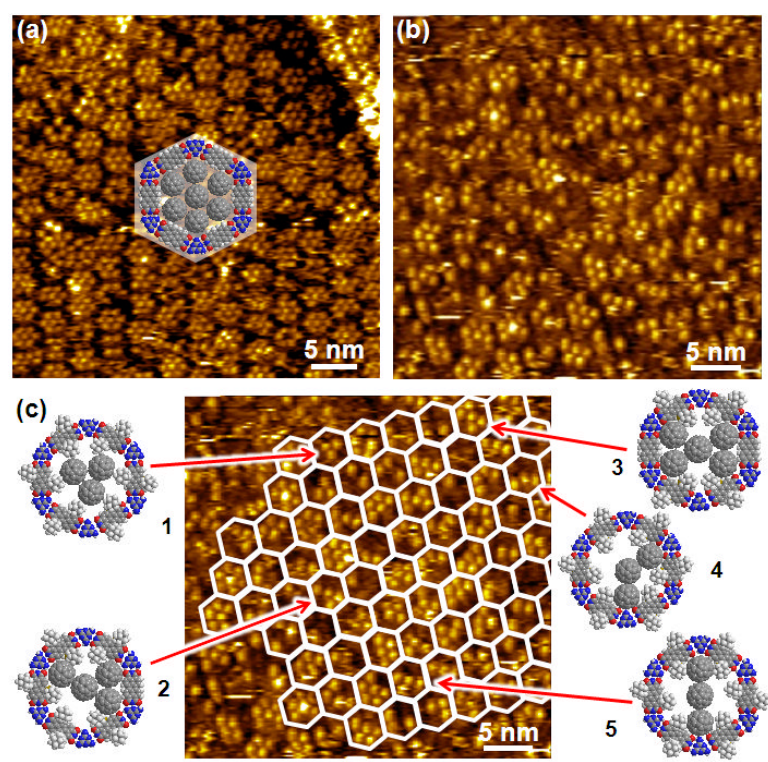

65 Fig. 5 STM images of $\mathrm{C}_{60}$ deposited into networks of PTCDI/melamine (a) and (Ad-S) $)_{2}-\mathrm{PTCDI} /$ melamine (b) on $\mathrm{Au}(111)$. Imaging parameters: $I$ $=28 \mathrm{pA}, V_{\text {bias }}=680 \mathrm{mV}$ (a) and $I=28 \mathrm{pA}, V_{\text {bias }}=340 \mathrm{mV}$ (b). c) same image as in b) with the honeycomb pattern of the network indicated as a guide to the eye. The models exemplify different arrangements of $\mathrm{C}_{60}$

70 molecules determined by the combination of (Ad-S $)_{2}-$ PTCDI enantiomers and missing adamantyl units. For details see text.

also is difficult to provide exact parameters for a respective imaging mode irrespective of general trends like the current dependent contrast.

75

\section{Fullerene adsorption}

While the size of the adamantyl unit allows for a seemless fit of $\mathrm{Ad}_{\mathrm{G}}$ molecules into the $\mathrm{Ad}_{\mathrm{N}}$ modified pore of the $(\mathrm{Ad}-\mathrm{S})_{2}-$ PTCDI/melamine network, this is expected to be very different ${ }_{80}$ for the significantly larger $\mathrm{C}_{60}$. To illustrate the effect of pore modification by the adamantyl moieties we show $\mathrm{C}_{60}$ adsorbed into an unmodified PTCDI/melamine network for comparison. As demonstrated in Fig. 5a, adsorption of the fullerenes from solution yields an arrangement of mostly seven molecules per 85 pore, identical to experiments where the $\mathrm{C}_{60}$ was deposited in a UHV environment. ${ }^{14}$

The analogous experiment carried out with the (Ad-S) $)_{2}-$ PTCDI /melamine network yields a very different picture. In contrast to the uniform clusters seen in Fig. 5a, there is a large variation with 90 regard to both the number of $\mathrm{C}_{60}$ molecules which ranges between 2 and 7 and geometry of the clusters as seen from Fig. 5b. Similar to the case shown in Fig. 3, the lack of uniform regular features makes it difficult to even identify the hexagonal network. However, the templating effect of the network becomes 95 obvious if the network pattern is overlaid as shown in Fig. 3c. Irrespective of their size and shape, all clusters are strictly confined to the hexagonal pores of the network.

There are two reasons for the diversity of clusters one of which is 
the defects in the network by missing $\operatorname{Ad}_{N}$ units as already discussed above (Fig. 3c,d). The occurrence of five or more $\mathrm{C}_{60}$ molecules in a pore can only be explained by this type of network imperfection. We note at this point that the number of missing ${ }_{5} \mathrm{Ad}_{\mathrm{N}}$ moieties seems to be significantly higher compared to the bare empty network. Recalling that the pure (Ad-S) $)_{2}-\mathrm{PTCDI}$ layer does not show any indication of missing adamantyl units, the number of defects seem to increase with increasing network modification. At this point we can only speculate as to the reason 10 for the enhanced proportion of defects but as the thioether link is clearly prone to cleavage then additional forces exerted by the adsorption of $\mathrm{C}_{60}$ would further increase the defect density.

The second reason for the occurrence of different $\mathrm{C}_{60}$ clusters is ascribed to the arrangement of the (Ad-S $)_{2}-\mathrm{PTCDI}$ molecules. As 15 indicated above two enantiomers of the PTCDI can be differentiated, unless the adamantyl units are located on the $\sigma_{2}$ mirror axis (see Fig. 1). Whether or not a fullerene can fit between two adjacent adamantyl units depends critically on the combination of enantiomers within a given pore. For two 20 neighbouring (Ad-S) $)_{2}-\mathrm{PTCDI}$ molecules of the same chirality there is insufficient space for a $\mathrm{C}_{60}$ to fit between the adamantyl units, whereas in the case of neighbouring adamantyl groups of opposing chirality there is sufficient space. Assuming that the $(+)$ and $(-)$ enantiomers are incorporated with equal probability, i.e. 25 this is not affected by the chirality of the neighbouring (Ad-S $)_{2}-$ PTCDI molecules, a number of adamantyl patterns becomes possible among which a regular pore structure consisting of only one enantiomer is the least likely of all configurations. For an enantiomerically pure pore geometry at most three $\mathrm{C}_{60}$ molecules 30 could be accommodated in the centre of the pore, as illustrated by model 1 in Fig. 5c. Indeed a configuration matching this geometry is infrequently observed. Compared to other $\mathrm{C}_{60}$ molecules which are located at the edge of the pore, the group of three is centred and, in accordance with the model, the corners of 35 the triangular arrangement of the fullerenes point towards the melamine molecules of the hydrogen-bonded network. Apart from this case of an enantiomerically pure pore geometry, where the $\mathrm{Ad}_{\mathrm{N}}$ units are arranged in a regular fashion, the combination of $(+)$ and $(-)$ enantiomers yields different spacings, thus giving 40 rise to different fullerene patterns. Structures 4 and 5 are examples where $\mathrm{C}_{60}$ molecules are located between two adamantyl units right at the melamine vortices of the network. The number of possible $\mathrm{C}_{60}$ arrangements is further increased by missing $\operatorname{Ad}_{\mathrm{N}}$ moieties. Any adjacent fullerenes located along a 45 pore edge require a missing adamantyl unit beside neighbouring (Ad-S) $)_{2}$-PTCDI molecules of opposite enantiomeric arrangement. These latter cases are the underlying structures 2 and 3 shown in Fig. 5c.

On the one hand, the variety of $\mathrm{C}_{60}$ configurations compiled in ${ }_{50}$ Fig. 5c, clearly demonstrate the opportunities for controlling the arrangement of guest molecules but also reveal the precision required for a full control over molecular geometries. The present investigation complements previous studies where PTCDI modified with two PrS or two bromo groups served as building

55 blocks in the bimolecular honeycomb structure formed on $\mathrm{Ag} / \mathrm{Si}(111)-\sqrt{3} \times \sqrt{3} \mathrm{R} 30^{\circ}{ }^{8 \mathrm{a}}$. Contrasting the influence of the adamantyl moieties, the bromo groups proved to be too small to affect the adsorption of $\mathrm{C}_{60}$ and similarly to the unsubstituted
PTCDI seven molecules were accommodated in the pores. The ${ }_{60} \mathrm{PrS}$ groups, on the other hand, had a partial blocking effect on the $\mathrm{C}_{60}$ adsorption although in an irregular manner. ${ }^{9}$ In the case of more rigid groups, such as benzoic acid moieties, ${ }^{17}$ a higher
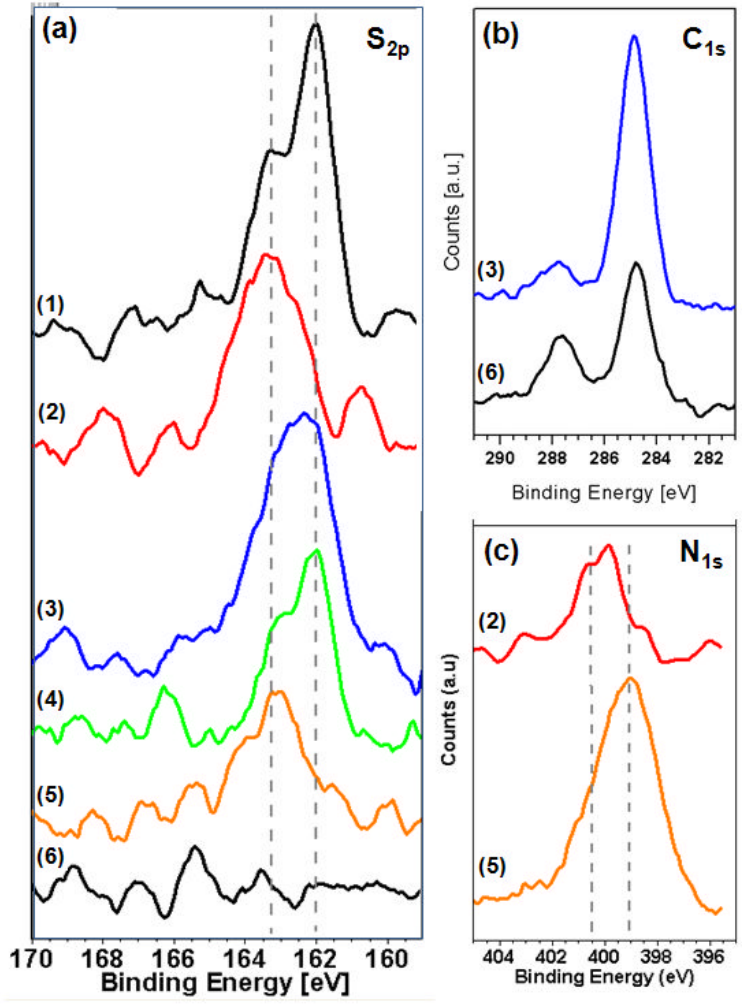

65 Fig. 6 XP spectra of PTCDI/melamine networks and an adamantanethiol $\mathrm{SAM}$ on $\mathrm{Au}(111) . \mathrm{S}_{2 \mathrm{p}}(\mathrm{a}), \mathrm{C}_{1 \mathrm{~s}}$ (b) and $\mathrm{N}_{1 \mathrm{~s}}$ regions are shown for pure AdSH-SAM (1), pure (Ad-S) $)_{2}-$ PTCDI monolayer (2), (Ad-S) $)_{2}-$ PTCDI $/$ Mel network with $\mathrm{AdSH}, \mathrm{Ad}_{\mathrm{G}}$, filled pores (3), PTCDI/Mel-network filled with $\mathrm{AdSH}, \mathrm{Ad}_{\mathrm{G}}$, filled pores (4), empty (Ad-S) ${ }_{2}-\mathrm{PTCDI} / \mathrm{Mel}$ 70 network (5), empty PTCDI/Mel network (6).

degree of control over molecular adsorption can be achieved. A similar approach for shaping pore structures has been explored with metal-organic coordination networks (MOCNs) based on $\mathrm{Fe}-1$,4-dicarboxylic benzoic acid and $\mathrm{Fe}$-1,2,4-tricarboxylic 75 benzoic acid on $\mathrm{Cu}(100){ }^{7}$ In these cases the cavities of both MOCNs accommodated one $\mathrm{C}_{60}$ molecule. Thus the additional - $\mathrm{COOH}$ side group of the 1,2,4-tricarboxylic benzoic acid molecules penetrating into the cavities did not block fullerene adsorption on the surface but instead resulted in an increased 80 host-guest interaction.

\section{XPS measurements}

For the chemical analysis of the supramolecular network and the adsorption of thiols into the pores, the sulfur signal is of particular interest as the binding energies differ for thiolates and ${ }_{85}$ thioethers. $^{42}$ The S $2 p$ spectra of the different systems are compiled in Fig. 6a. Spectrum 1 shows a uniform adamantane thiol SAM with a peak around $162 \mathrm{eV}$ and a shoulder at 163.3 $\mathrm{eV}$, assigned to the $2 \mathrm{p}_{3 / 2}$ and $2 \mathrm{p}_{1 / 2}$ doublet at binding energies characteristic of thiolates. For the pure (Ad-S $)_{2}-$ PTCDI 90 monolayer (spectrum 2) the peak appears at $163.3 \mathrm{eV}$ as expected 
for a thioether species. The PTCDI/melamine network with the pores filled by adamantane thiol yields a spectrum (4) which is identical to the uniform adamantane thiol SAM (1) apart from the lower intensity due to the reduction in area of the SAM by the 5 hydrogen-bonded network. Analogously, the combination of (AdS) ${ }_{2}$-PTCDI with melamine to the network (5) yields a position of the sulfur peak identical to that of the uniform (Ad-S) $)_{2}-\mathrm{PTCDI}$ layer. Again the network structure reduces the density and, thus, intensity of the signal. Finally, the network-SAM hybrid structure 10 where the adamantane thiolate molecules filling the network pores and the thioether species of the network coexist yields a superposition of both signals as reflected by a position of the sulfur peak in between the thiolate at $162 \mathrm{eV}$ and the thioether at $163.3 \mathrm{eV}$. For completeness the signal of a PTCDI-melamine 15 network is shown (6) to illustrate the signal/noise ratio.

As far as the other elements are concerned there are, as expected, no significant differences. The $\mathrm{C} 1 \mathrm{~s}$ spectrum consists, in all cases, of a peak around $284.5 \mathrm{eV}$ and a second peak of lower intensity around $287.5 \mathrm{eV}$ which originate from the 20 aliphatic/aromatic carbon and carbonyl species, respectively. The AdSH filled (Ad-S $)_{2}-\mathrm{PTCDI} /$ melamine network and the PTCDI/melamine network are shown as illustrative examples with the biggest difference between systems. Comparing both spectra, the peak is at $284.5 \mathrm{eV}$ is higher for the pore filled 25 network due to the additional AdSH molecules. Conversely, the carbonyl peak is weaker than in the PTCDI/melamine case due to the attenuation of the photoelectrons by the AdSH layer. We note that the attenuation is amplified due to the geometry of the experiment as the photoelectrons emitted an angle of $53^{\circ}$ from the 30 surface normal were measured.

The $\mathrm{N}$ 1s spectra are also very similar apart from the difference between layers consisting of (Ad-S) $)_{2}-\mathrm{PTCDI}$ molecules only and of network combining (Ad-S $)_{2}-$ PTCDI and melamine. Spectra of the former are characterised by a single nitrogen peak at $\sim 400 \mathrm{eV}$ 35 from the imide nitrogen whereas in the network spectra the signal is dominated by the melamine nitrogen which peaks at $399 \mathrm{eV}$. In accordance with the higher nitrogen content of melamine the signal is higher compared to pure (Ad-S) $)_{2}-\mathrm{PTCDI}$ despite the more open structure of the network.

\section{${ }_{40}$ Conclusions}

We have demonstrated that it is possible to introduce a bulky $3 \mathrm{D}$ substituent as pore modifier of the PTCDI-melamine surfacebased hydrogen bonded network. The influence of the large adamantyl thioether moieties on the adsorption of the host 45 molecules is sensitively dependent on the size of the host molecules. While adamantane thiol fits seamlessly into the modified pores, the situation is pronouncedly different for fullerenes due to the surface-based chirality of the (Ad-S $)_{2}-$ PTCDI molecule. The statistical combination of the enantiomers 50 gives rise to different templating pore geometries, thus yielding different arrangements of $\mathrm{C}_{60}$ molecules. Partial cleavage of the adamantyl thioether substituents upon preparation of the bimolecular (Ad-S $)_{2}-\mathrm{PTCDI} /$ melamine array further increases the number of possible fullerene arrangements. Even though at 55 present the different patterns of fullerene guest molecules are realised in a statistical fashion, the system presented here demonstrates the opportunities for the arrangement of guest entities through modification of pore geometries We continue to pursue our understanding of the formation of surface-based ${ }_{60}$ hydrogen-bonded networks in order to accomplish full control over the pore design.

\section{Acknowledgements}

This work was supported by the UK Engineering Physical Sciences Research Council (EPSRC). NRC gratefully 65 acknowledges the receipt of a Royal Society Leverhulme Trust Senior Research Fellowship. MTR acknowledges the financial support of the Emil Aaltonen Foundation and the Academy of Finland.

\section{Notes and references}

$7^{2}{ }^{a}$ EaStCHEM School of Chemistry, University of St Andrews, North Haugh, St Andrews KY16 9ST, United Kingdom. Fax: 441334 463808; Tel: 441334 463800;E-mail:mb45@st-andrews.ac.uk ${ }^{b}$ present address: Laboratory of Inorganic Chemistry, Department of Chemistry, University of Helsinki, P. O. Box 55, FI-00014 University of 75 Helsinki, Finland

${ }^{b}$ School of Chemistry, University of Nottingham, University Park, Nottingham NG7 2RD, United Kingdom. Fax: 441159 513563; Tel:44 1159 513505; E-mail: Neil.Champness@nottingham.ac.uk

80

1. F. Cicoira, C. Santato and F. Rosei, Top. Curr. Chem., 2008, 285, 203-267.

2. J. V. Barth, Surf. Sci., 2009, 603, 1533-1541.

85 3. J. A. A. W. Elemans, S. B. Lei and S. De Feyter, Angew. Chem. Int. Ed., 2009, 48, 7298-7332.

4. S. Furukawa and S. De Feyter, Top Curr Chem, 2009, 287, 87-133.

5. T. Kudernac, S. B. Lei, J. A. A. W. Elemans and S. De Feyter, Chem. Soc. Rev., 2009, 38, 3505-3505.

90 6. N. Lin, S. Stepanow, M. Ruben and J. V. Barth, Top Curr Chem, 2009, 287, 1-44.

7. S. Stepanow, M. Lingenfelder, A. Dmitriev, H. Spillmann, E. Delvigne, N. Lin, X. B. Deng, C. Z. Cai, J. V. Barth and K. Kern, Nature Materials, 2004, 3, 229-233.

95 8. A. G. Slater, P. H. Beton and N. R. Champness, Chem. Sci., 2011, 2, 1440-1448.

9. L. M. A. Perdigao, A. Saywell, G. N. Fontes, P. A. Staniec, G. Goretzki, A. G. Phillips, N. R. Champness and P. H. Beton, Chem. Eur. J., 2008, 14, 7600-7607.

100 10. L. M. A. Perdigao, E. W. Perkins, J. Ma, P. A. Staniec, B. L. Rogers, N. R. Champness and P. H. Beton, J. Phys. Chem. B, 2006, 110, 12539-12542.

11. F. Silly, A. Q. Shaw, K. Porfyrakis, G. A. D. Briggs and M. R. Castell, Appl. Phys. Lett., 2007, 91, -

105 12. A. Saywell, G. Magnano, C. J. Satterley, L. M. A. Perdigao, N. R. Champness, P. H. Beton and J. N. O'Shea, J. Phys. Chem. C, 2008, 112, 7706-7709.

13. J. A. Theobald, N. S. Oxtoby, N. R. Champness, P. H. Beton and T. J. S. Dennis, Langmuir, 2005, 21, 2038-2041.

110 14. J. A. Theobald, N. S. Oxtoby, M. A. Phillips, N. R. Champness and P. H. Beton, Nature, 2003, 424, 1029-1031.

15. R. Madueno, M. T. Raisanen, C. Silien and M. Buck, Nature, 2008 , 454, 618-621.

16. C. Silien, M. T. Raisanen and M. Buck, Angew. Chem. Int. Ed., 2009, $115 \quad$ 48, 3349-3352.

17. C. Silien, M. T. Raisanen and M. Buck, Small, 2010, 6, 391-394.

18. A. G. Phillips, L. M. A. Perdigao, P. H. Beton and N. R. Champness, Chem. Commun., 2010, 46, 2775-2777.

19. A. J. Pollard, E. W. Perkins, N. A. Smith, A. Saywell, G. Goretzki, 120 A. G. Phillips, S. P. Argent, H. Sachdev, F. Muller, S. Hufner, S. Gsell, M. Fischer, M. Schreck, J. Osterwalder, T. Greber, S. Berner, 
N. R. Champness and P. H. Beton, Angew. Chem. Int. Ed., 2010, 49, 1794-1799.

20. D. Bonifazi, O. Enger and F. Diederich, Chem. Soc. Rev., 2007, 36, 390-414.

5 21. A. A. R. Watt, M. R. Sambrook, K. Porfyrakis, B. W. Lovett, H. El Mkami, G. M. Smith and G. A. D. Briggs, Chem. Commun., 2006, 1944-1946.

22. S. J. H. Griessl, M. Lackinger, F. Jamitzky, T. Markert, M. Hietschold and W. M. Heckl, J. Phys. Chem. B, 2004, 108, 1155611560 .

23. G. B. Pan, J. M. Liu, H. M. Zhang, L. J. Wan, Q. Y. Zheng and C. L. Bai, Angew. Chem. Int. Ed., 2003, 42, 2747-2751.

24. P. A. Staniec, L. M. A. Perdigao, A. Saywell, N. R. Champness and P. H. Beton, ChemPhysChem, 2007, 8, 2177-2181.

15 25. M. Stohr, M. Wahl, H. Spillmann, L. H. Gade and T. A. Jung, Small, 2007, 3, 1336-1340.

26. S. Yoshimoto, Y. Honda, O. Ito and K. Itaya, J. Am. Chem. Soc., 2008, 130, 1085-1092.

27. D. Bonifazi, H. Spillmann, A. Kiebele, M. de Wild, P. Seiler, F. Y.

20 Cheng, H. J. Guntherodt, T. Jung and F. Diederich, Angew. Chem. Int. Ed., 2004, 43, 4759-4763.

28. E. Mena-Osteritz and P. Bauerle, Adv. Mater., 2006, 18, 447-451.

29. H. Spillmann, A. Kiebele, M. Stohr, T. A. Jung, D. Bonifazi, F. Y. Cheng and F. Diederich, Adv. Mater., 2006, 18, 275-279.

25 30. A. Kiebele, D. Bonifazi, F. Y. Cheng, M. Stohr, F. Diederich, T. Jung and H. Spillmann, ChemPhysChem, 2006, 7, 1462-1470.

31. M. Li, K. Deng, S. B. Lei, Y. L. Yang, T. S. Wang, Y. T. Shen, C. R. Wang, Q. D. Zeng and C. Wang, Angew. Chem. Int. Ed., 2008, 47, 6717-6721.

30 32. L. Sanchez, R. Otero, J. M. Gallego, R. Miranda and N. Martin, Chem. Rev., 2009, 109, 2081-2091.

33. A. A. Dameron, T. J. Mullen, R. W. Hengstebeck, H. M. Saavedra and P. S. Weiss, J. Phys. Chem. C, 2007, 111, 6747-6752.

34. A. Marchenko and J. Cousty, Surf. Sci., 2002, 513, 233-237.

35 35. J. A. Gardener, G. A. D. Briggs and M. R. Castell, Phys. Rev. B, 2009, 80, 235434.

36. R. Fasel, M. Parschau and K.-H. Ernst, Nature, 2006, 439, 449-452.

37. J. Ziroff, P. Gold, A. Bendounan, F. Forster and F. Reinert, Surf. Sci., 2009, 603, 354-358.

40 38. J. C. Russell, M. O. Blunt, G. Goretzki, A. G. Phillips, N. R. Champness and P. H. Beton, Langmuir, 2010, 26, 3972-3974.

39. C. Jung, O. Dannenberger, Y. Xu, M. Buck and M. Grunze, Langmuir, 1998, 14, 1103-1107.

40. C. J. Zhong, R. C. Brush, J. Anderegg and M. D. Porter, Langmuir, 45 1999, 15, 518-525.

41. H. Takiguchi, K. Sato, T. Ishida, K. Abe, K. Yase and K. Tamada, Langmuir, 2000, 16, 1703-1710.

42. T. Weidner, N. Ballav, U. Siemeling, D. Troegel, T. Walter, R. Tacke, D. G. Castner and M. Zharnikov, J. Phys. Chem. C, 2009, 113, 19609-19617. 